\title{
The effects of transient attention on spatial resolution and the size of the attentional cue
}

\author{
YAFFA YeSHURUN \\ University of Haifa, Haifa, Israel \\ AND \\ Marisa Carrasco \\ New York University, New York, New York
}

\begin{abstract}
It has been shown that transient attention enhances spatial resolution, but is the effect of transient attention on spatial resolution modulated by the size of the attentional cue? Would a gradual increase in the size of the cue lead to a gradual decrement in spatial resolution? To test these hypotheses, we used a texture segmentation task in which performance depends on spatial resolution, and systematically manipulated the size of the attentional cue: A bar of different lengths (Experiment 1) or a frame of different sizes (Experiments 2-3) indicated the target region in a texture segmentation display. Observers indicated whether a target patch region (oriented line elements in a background of an orthogonal orientation), appearing at a range of eccentricities, was present in the first or the second interval. We replicated the attentional enhancement of spatial resolution found with small cues; attention improved performance at peripheral locations but impaired performance at central locations. However, there was no evidence of gradual resolution decrement with large cues. Transient attention enhanced spatial resolution at the attended location when it was attracted to that location by a small cue but did not affect resolution when it was attracted by a large cue. These results indicate that transient attention cannot adapt its operation on spatial resolution on the basis of the size of the attentional cue.
\end{abstract}

Covert spatial attention allows us to select visual information at a cued location without eye movements. It can be voluntarily allocated to a given location according to endogenous goals - sustained attention — or involuntarily allocated, in a fast reflexive manner, to a stimulus that appears suddenly in the visual field-transient attention (see, e.g., Jonides, 1981; H. J. Müller \& Rabbitt, 1989; Nakayama \& Mackeben, 1989; Posner, 1980; Yantis, 1996). A large body of evidence has demonstrated that attending a specific location improves performance on a variety of tasks, including early vision tasks, in which performance depends on contrast sensitivity (e.g., Carrasco, Penpeci-Talgar, \& Eckstein, 2000; Lu \& Dosher, 1998; Pestilli \& Carrasco, 2005). It has also been shown that transient attention can aid performance by enhancing spatial resolution at the attended location (e.g., Carrasco, Williams, \& Yeshurun, 2002; Golla, Ignashchenkova, Haarmeier, \& Thier, 2004; Yeshurun \& Carrasco, 1998, 1999, 2000). Because spatial resolution is defined as our ability to see and resolve small details in a visual scene, this implies that attention enhances our ability to detect fine details at the attended location. In most cases, it is advantageous for the system to have enhanced resolution, but in some cases enhanced resolution can hamper performance (e.g., Carrasco, Loula, \& Ho, 2006; Talgar \& Carrasco, 2002; Yeshurun \& Carrasco, 1998, 2000). The goal of this study was to investigate the adaptability of transient attention regarding spatial resolution: Can transient attention modulate its effect on spatial resolution when it is cued to attend larger areas? In particular, we investigated whether the scale of the information that attracts attention (the cue size) can modulate the effects of transient attention on spatial resolution at the attended location.

Previous studies have provided evidence for the hypothesis that transient attention can enhance spatial resolution. For instance, directing transient attention to the target location improves performance in both acuity and hyperacuity tasks, even when a suprathreshold target is presented without distractors. When a small peripheral cue $\left(\leq 1^{\circ}\right)$ indicates the location of the upcoming target, observers are able to discriminate the location of a smaller gap appearing on a Landolt square, and to identify smaller horizontal offsets of a Vernier target, than when they have no prior information regarding the target location (Yeshurun $\&$ Carrasco, 1999). The same pattern of results is found whether or not a mask follows a target - that is, whether or not all sources of added external noise have been eliminated (Carrasco et al., 2002). Similarly, in a visual search task, the typical decrement in performance that occurs as the target is presented at farther peripheral locations is significantly reduced when observers direct their attention to the target location (Carrasco \& Yeshurun, 1998). 
Because this eccentricity effect is attributed to the poorer spatial resolution at the periphery (see, e.g., Carrasco, Evert, Chang, \& Katz, 1995; Carrasco \& Frieder, 1997; Carrasco, McLean, Katz, \& Frieder, 1998), the ability of the peripheral cue to reduce this performance decrement suggests that attention can reduce resolution differences between the fovea and the periphery.

We have performed a crucial test of the resolution hypothesis by exploring the effects of transient attention on a texture segregation task in which performance is diminished by heightened resolution (Yeshurun \& Carrasco, 1998). If attention enhances resolution, performance at the attended location should be impaired rather than improved. The texture segregation task involves the detection of a texture target embedded in a background of an orthogonal orientation (Figure 1). Observers' performance in this task does not peak when the target is presented at foveal locations, where resolution is highest. Instead, performance peaks at midperipheral locations and drops when the target appears at more central or farther peripheral locations. The finding that performance in this texture segregation task drops at central locations - the central performance drop - is attributed to a mismatch between the average size of spatial filters at the fovea and the scale of the texture (see, e.g., Gurnsey, Pearson, \& Day, 1996; Kehrer, 1997). There is ample evidence that we process visual stimuli by means of parallel spatial filters. These are low-level analyzers tuned to a specific band of spatial frequency and orientation (e.g., De Valois \& De Valois, 1988; Graham, 1989; Phillips \& Wilson, 1984). The size of these filters at the fovea is too small for the scale of the texture, resulting in spatial resolution that is too high for the task. At more peripheral regions, the filters' average size increases gradually, presumably reaching optimal size around the area of peak performance. At farther locations, the filters are too big, and their low resolution limits performance (e.g., Gurnsey et al., 1996; Joffe \& Scialfa, 1995; Kehrer, 1989, 1997; Potechin \& Gurnsey, 2003).

We hypothesized that if attention enhances spatial resolution, attending to the target location should enhance performance in the periphery, where resolution is too low, but should impair performance at the fovea, where resolution is already too high for the task. To test this prediction, we combined peripheral cues with the texture segregation task (Yeshurun \& Carrasco, 1998). On the cued trials, a small peripheral cue $\left(\leq 1^{\circ}\right)$ indicated the target location prior to appearance of the target, inducing observers to focus their transient attention on the target location, without having time to move their eyes to that location. Such a peripheral cue is considered to capture attention in a stimulus-driven, "automatic" manner (see, e.g., Jonides, 1981; H. J. Müller \& Rabbitt, 1989; Posner, 1980; Yantis, 1996). On the rest of the trials, a pair of lines appearing above and below the entire display indicated that the target was equally likely to
Exp. 1

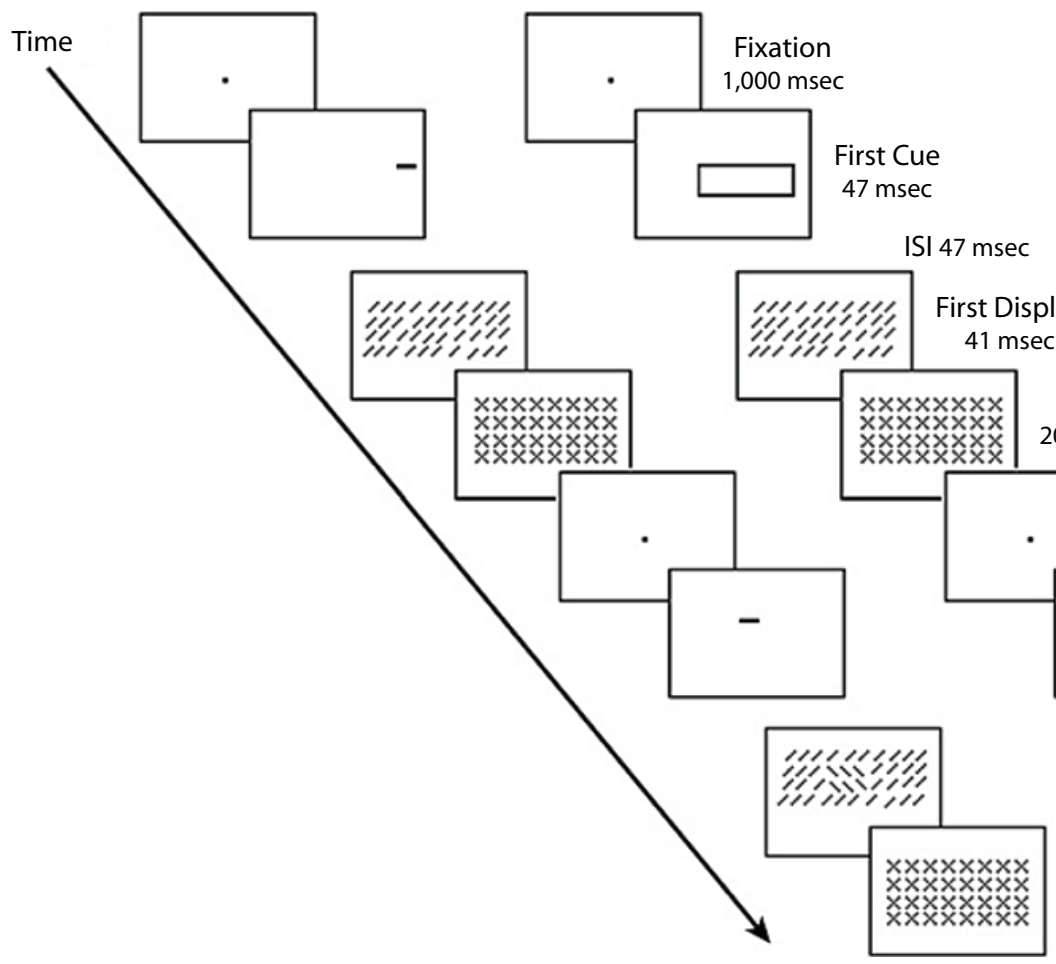

Exp. 2 
appear at any location. As expected, accuracy was higher when the observers could focus their attention on peripheral target locations, but when the target appeared at central locations, the peripheral cue lowered performance. Hence, attending the target location improved performance at peripheral locations, where resolution was too low for the scale of the texture, but impaired performance at central locations, where resolution was already too high, resulting in a central attentional impairment. It is worth noting that this counterintuitive impairment is not predicted by any model of attention based on external noise reduction, reduction of spatial uncertainty, or decision-making processes; all of these models predict an attentional benefit at all locations. Thus, this finding sheds light on the nature of the attentional mechanism by lending strong support to the hypothesis that attention enhances spatial resolution at the attended location (Yeshurun \& Carrasco, 1998). To further probe this resolution hypothesis, we enlarged the scale of the texture. Consistent with previous findings (Gurnsey et al., 1996; Joffe \& Scialfa, 1995), increasing the texture scale moved the performance peak to farther eccentricities. Moreover, the central attentional impairment changed as a function of texture scale: Performance was impaired in a larger range of central retinal locations as the scale of the texture increased. That is, for the same target eccentricity, we found either an attentional improvement or impairment depending on the scale of the texture (Talgar \& Carrasco, 2002; Yeshurun \& Carrasco, 1998). This finding rules out the possibility that attention is unable to enhance processing of foveal and parafoveal stimuli in this task.

Similar central attentional impairments have been obtained when the texture is presented along the vertical rather than the horizontal meridian (Talgar \& Carrasco, 2002), when observers selectively adapt to different spatial frequencies before performing the texture segmentation task (Carrasco et al., 2006), and when the first- and second-order contents of the textures are manipulated (Yeshurun \& Carrasco, 2000). These various experimental conditions demonstrate that such effects are robust and can be generalized to textures of different natures.

The findings that attention enhances resolution are consistent with neurophysiological studies suggesting that attention contracts the cell's receptive field around the attended stimulus (see, e.g., Desimone \& Duncan, 1995; Luck, Chelazzi, Hillyard, \& Desimone, 1997; Moran \& Desimone, 1985). These studies have found that the neural response to a stimulus that normally elicits strong activity is greatly reduced when this "effective stimulus" is not attended but a "noneffective stimulus" in the same receptive field is attended (for reviews, see Reynolds \& Chelazzi, 2004; Reynolds \& Desimone, 1999).

By enhancing resolution, attention allows us to better resolve the various details in the environment. This would often be advantageous, because many of our everyday life tasks require heightened resolution. However, when a more global assessment of the display is required - for example, when one is appreciating an impressionist painting or is more interested in seeing the forest than the individual trees - enhancing resolution is not the optimal strategy (as evidenced by the central attentional impairment). Similarly, a high-resolution analysis of the scene would not be optimal when information of high spatial resolution is not present in the visual scene, as when we have to navigate through the world under less than optimal atmospheric conditions (e.g., when it is foggy or hazy). In situations in which heightened resolution is not optimal, can transient attention adapt its operation, enhancing or decreasing resolution to optimize performance? The purpose of this study was to investigate whether transient attention is functionally flexible and adaptable to the characteristics of the environment. We explored the possibility that the attentional effect on spatial resolution depends on the scale of the attention-attracting information. Specifically, we asked whether a gradual increase in the size of the attentional cue would lead to a gradual resolution decrement.

The adaptability of visual attention has been studied extensively. Several studies have explored the ability of visual attention to shift between global and local structural levels of hierarchical stimuli. In most studies, the attentional manipulation was accomplished by varying the expectations regarding the relevant level of analysis, and their results suggest that attention can be allocated categorically to a structural level, either global or local (e.g., Hübner, 2000; Kinchla, Solis-Macias, \& Hoffman, 1983; Lamb \& Yund, 1996; Robertson, Egly, Lamb, \& Kerth, 1993; Shulman \& Wilson, 1987; Ward, 1982). Although some of these studies used attentional cues on a trial-by-trial basis (e.g., Hübner, 2000; Robertson et al., 1993), the cues predicted the target's structural level rather than location, and therefore did not manipulate spatial attention.

More relevant to our present goal are studies that did employ attentional cues to predict the target location but also varied the size of the cues in order to explore their differential effects on performance (e.g., Castiello \& Umiltà, 1990; Eriksen \& St. James, 1986; Eriksen \& Yeh, 1985; LaBerge, Brown, Carter, Bash, \& Hartley, 1991). For instance, in a visual search task, Greenwood and Parasuraman (2004) used spatial cues of different sizes, on the assumption that larger cues would encourage a more diffuse, sustained attention allocation and that small cues would encourage a more focused mode of sustained attention. They found that search reaction times (RTs) were faster as the size of the cue decreased. However, when observers could not predict the size and location of the target, their performance was superior with large rather than small cues. On the basis of these results, the authors concluded that attentional scaling is optimized for task demand - that is, narrow for small targets, broad when target size and location are unpredictable. Similarly, in an fMRI study of sustained attention, N. G. Müller and colleagues (N. G. Müller, Bartelt, Donner, Villringer, \& Brandt, 2003) analyzed neural activity for target detection with a preceding central cue that indicated whether a small, medium, or large region had to be attended. Their analysis demonstrated that as the size of the attended region increased, the extent of activated retinotopic visual cortex also increased. However, consistent with the idea of limited resources, the level of neural activity in a given subregion decreased. 
The size of the attended region has also been manipulated with dual tasks. Goto, Toriu, and Tanahashi (2001) measured contrast sensitivity functions while observers simultaneously performed an instantaneous judgment task, which was designed to control the size of the attended region. Goto et al. found higher sensitivity for higher spatial frequencies (over 3 cycles/degree) only under the narrowly-attended-region condition. Likewise, when the size of the attended region was manipulated using different dual tasks, vernier acuity increased when attention was narrowly focused on a foveal target but not when it was broadly spread (Balz \& Hock, 1997), and a broad spread of attention affected self-organized motion patterns in a manner consistent with the activation of large filters (Hock, Balz, \& Smollon, 1998).

These various studies are consistent with the idea that the larger the zoom lens, the lower its resolution (see, e.g., Eriksen, 1990). Although these studies manipulated the more controlled, slower component of spatial attentionsustained attention - and notwithstanding the differences between sustained and transient attention with regard to perceptual effects (e.g., Ling \& Carrasco, 2006), temporal characteristics (e.g., Nakayama \& Mackeben, 1989), degree of automaticity (e.g., Yantis, 1996), and neural mechanisms (e.g., Corbetta \& Shulman, 2002; Rosen et al., 1999), these findings suggest that manipulating the size of attentional cues designed to attract transient attention may also have a differential effect on performance. Specifically, small cues may lead to resolution enhancement, and large cues to resolution decrement. To test this hypothesis, we employed two different attentional cues and systematically manipulated their size: In Experiment 1, a horizontal bar of different lengths appeared above the target region, and in Experiments 2 and 3, a frame of different sizes surrounded the target region. The largest cue surrounded the entire display and conveyed no information regarding the target location. This noninformative cue served as the comparison with which performance with smaller cues was compared. The cues were combined with a texture segmentation task similar to that used by Yeshurun and Carrasco (1998); this task involved the detection of a texture orientation target appearing at various eccentricities in a background with an orthogonal orientation (Figure 1). Were transient attention able to modulate its effect on spatial resolution as a function of the cue size - so that the larger the cue, the lower the resolution-performance at central locations should gradually improve and performance at peripheral locations gradually deteriorate as cue size increases. Moreover, as cue size increases, the eccentricity at which performance peaks should gradually shift to nearer eccentricities, reflecting the gradual decrease in resolution. For the noninformative condition, performance should peak at the nearest eccentricity because the noninformative cue designates the largest area - the whole display. Alternatively, if transient attention does not alter its operation according to the size of the attentional cue, its effect on spatial resolution will not change in a gradual fashion with changes in cue size.

\section{EXPERIMENT 1}

To test the hypothesis that the effect of transient attention on spatial resolution will change as a function of cue size, we employed a two-interval forced choice task (2IFC; Figure 1). A trial consisted of two temporal intervals, each containing a texture display preceded by a cue. The texture target appeared equally often in each temporal interval and at any of several possible eccentricities within the texture background. On the informative trials, the cue was a horizontal bar of a certain size, indicating the onset of the upcoming texture and the region in which the target would appear. On the noninformative trials, the cue did not convey information regarding the target location. The observers were instructed to report whether the texture target was present in the first or the second interval. This 2IFC task ensured that attention-related response biases would be avoided: The cue indicated that if the target were to be present, it could only appear within the cued region (100\% valid cue), but the cue did not signal which of the two intervals was more likely to contain the target, because both displays in a trial were preceded by a cue.

\section{Method}

Observers. Twenty-two undergraduate students from the University of Haifa participated as observers in this experiment; all had normal or corrected-to-normal vision and were naive as to the purpose of the study.

Stimuli and Apparatus. The stimuli were presented using PsyScope (Cohen, MacWhinney, Flatt, \& Provost, 1993) on the 21-in. monitor of a Power Mac G4 computer (resolution 1,280 × 1,024 pixels, $85 \mathrm{~Hz})$. The texture display was presented on a white screen $\left(87 \mathrm{~cd} / \mathrm{m}^{2}\right)$, and it included a $2^{\circ} \times 2^{\circ}$ texture target composed of $3 \times 3$ black lines (each subtending $0.1^{\circ} \times 0.7^{\circ}$ and oriented at $45^{\circ}$ or $135^{\circ}$ ) embedded in a $5^{\circ} \times 28^{\circ}$ texture background composed of 287 black lines ( 7 rows $\times$ 41 columns) whose orientation was orthogonal to that of the target. The target was centered at one of 15 possible locations along the horizontal meridian, corresponding to seven eccentricities (one at the center and six on either side of fixation: $0^{\circ}, 0.7^{\circ}, 1.5^{\circ}, 3^{\circ}, 5^{\circ}, 7.5^{\circ}, 11.5^{\circ}$ ), appearing equally often at each eccentricity. The mask texture was composed of 287 black " $X$ " elements ( 7 rows $\times 41$ columns). The attentional cue was a green $\left(43 \mathrm{~cd} / \mathrm{m}^{2}\right)$ horizontal bar appearing $0.3^{\circ}$ above the region of texture in which the target could appear (i.e., 2.3 $3^{\circ}$ above the central horizontal meridian). The size of the bar varied systematically between $1\left(0.2^{\circ} \times 0.7^{\circ}\right.$ - the width of 1 column $)$ and $15\left(0.2^{\circ} \times\right.$ $10^{\circ}$ - the width of 15 columns), for a total of five possible cue sizes $(1,3,6,9$, or 15). The noninformative cue included two long, green $\left(43 \mathrm{~cd} / \mathrm{m}^{2}\right)$ horizontal lines $\left(0.2^{\circ} \times 28^{\circ}\right)$ appearing $0.5^{\circ}$ above and below the entire display.

Procedure. Each temporal interval of the 2IFC task began with a 1,000-msec central fixation dot (Figure 1), which was followed by a $47-\mathrm{msec}$ cue and a $47-\mathrm{msec}$ interstimulus interval (ISI). Two thirds or three fourths of the total trials (cues of Sizes 1-6 or 9-15, respectively) were informative trials. On these trials, in the interval containing the target, the cue appeared above the texture region that included the target patch. Cue Sizes 1 and 3 indicated the target location with $100 \%$ certainty. With Cue Sizes $6-15$, the exact location of the target within the cued region was chosen randomly to prevent narrow focusing of attention on an expected location. Thus, the larger the cue, the greater the degree of uncertainty, though the degree of uncertainty involved with the noninformative cue was always higher (e.g., with the noninformative cue, the target could appear anywhere within the display - 41 texture columns - whereas with the largest informative cue - Size 15- the target could appear only within 15 texture columns, or about one third of the entire display). In the in- 
terval without a target, the cue appeared above a randomly chosen texture region. On the remaining trials (the noninformative trials), a noninformative cue indicated, in both intervals, that the target had equal probability of appearing at any of the possible locations. The durations of the cue and the ISI were chosen to ensure that the time between cue onset and texture onset was optimal for transient attention to focus on the cued region (see, e.g., Cheal \& Lyon, 1992; Nakayama \& Mackeben, 1989). After the ISI, the texture was displayed for an average of $41 \mathrm{msec}$. The duration of the texture presentation was set individually to avoid floor or ceiling effects, but it did not exceed $82 \mathrm{msec}$, to ensure that eye movements could not take place between cue onset and texture offset (e.g., Mayfrank, Kimmig, \& Fischer, 1987). Immediately following the texture, a $200-\mathrm{msec}$ mask was presented. The cues of different sizes were blocked, so that each experimental block included two types of cues: an attentional, informative cue of a specific size and a noninformative cue. Each observer performed some practice trials (between 100 and 160) and then 1,120 experimental trials divided into five blocks. The order of trials within a block and the order of blocks within the experiment were randomized.

\section{Results and Discussion}

To test whether the effect of cue type (informative vs. noninformative) varied as a function of cue size and target eccentricity, the data were subjected to a within-observers three-way ANOVA (cue type $\times$ cue size $\times$ target eccentricity). A marginally significant three-way interaction $[F(24,504)=1.44, p<.067]$ indicated a differential attentional effect for the different cue sizes, which appears more clearly when the data are inspected separately for the different cue sizes (Figure 2). As in several studies examining texture segregation across eccentricities (e.g., Joffe \& Scialfa, 1995; Kehrer, 1989; Yeshurun \& Carrasco, 1998, 2000), the data were fitted to second-order polynomials for the noninformative and informative conditions at each cue size. Given that the noninformative trials in the different experimental blocks were identical, that fewer noninformative than informative trials took place per block, and that no significant difference was found between noninformative trials blocked with different cue sizes, we combined the data of all noninformative trials across block conditions. The data were further analyzed with a withinobservers two-way ANOVA (cue type $\times$ target eccentricity) performed separately for each cue size. As is evident in Figure 2, we replicated our previous finding: A significant cue type $\times$ target eccentricity interaction emerged for the smallest attentional cue, showing that attention degraded performance at central locations and improved it at peripheral locations $[F(6,126)=6.91, p<.0001]$. Although a significant interaction also emerged for Cue Size 9, it only reflected an attentional cost at the central locations, without a distinctive benefit at the periphery $[F(6,126)=2.12$, $p<.05]$. The explanation for this interaction at Cue Size 9 is not clear, and because this interaction was not replicated in the other two experiments it will not be discussed further. A significant interaction was not found with any other cue size. In fact, the only other significant effect found was a main effect of cuing condition at Cue Size 3, in which accuracy was higher in the informative than in the noninformative condition $[F(1,21)=5.62, p<.05]$. An analysis of correct RTs confirmed that no speed-accuracy trade-offs occurred.

In addition, we tested whether large cues gradually shift the performance peak to nearer eccentricities, with the performance peak of the noninformative condition being at the nearest eccentricity. To that end, we estimated (via second-order polynomials) the eccentricity at which performance peaked for each of the three large-cue conditions (i.e., Cue Sizes 6, 9, and 15, in which the informative cue was larger than the target) and for the noninformative condition (see Table 1). As can be seen, there was no consistent difference in the peak eccentricity of the different cues, including the noninformative cue.

In summary, as is the case with sustained attention (see, e.g., Castiello \& Umiltà, 1990; Eriksen \& St. James, 1986; Goto et al., 2001; Greenwood \& Parasuraman, 2004; Hock et al., 1998; LaBerge et al., 1991; N. G. Müller et al., 2003), a differential effect was found for the different cue sizes, but in the present experiment it mainly reflected an attentional effect for the small cue sizes $(1,3)$ and no effect for the larger cues (6-15). No gradual change in performance occurred with increasing cue size. These findings indicate that in this texture segmentation task, transient attention exerted its effects on spatial resolution only when it was directed to a small region by a small cue. There was no evidence that transient attention could flexibly lower resolution when attracted to a broader spatial region by large cues. Alternatively, this pattern of results might be due to the specific attentional cue used in this experiment - a single bar above the target region. The advantage of employing this cue was that the

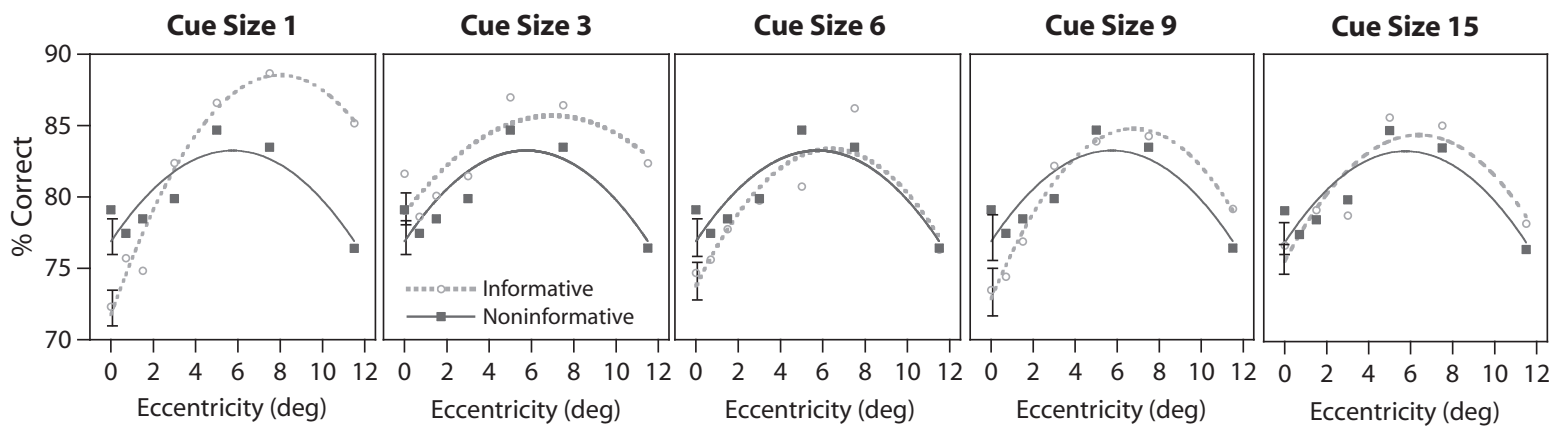

Figure 2. Observers' accuracy in Experiment 1 as a function of cue size and target eccentricity. Error bars correspond to one averaged standard error. 
smallest bar (Cue Size 1) was identical to the attentional cue that yielded an attentional effect on spatial resolution (e.g., Carrasco et al., 2006; Talgar \& Carrasco, 2002; Yeshurun \& Carrasco, 1998), thus allowing a direct comparison of present and previous findings. However, the single bar might not have led to a resolution decrement because it may not have successfully encouraged observes to utilize the information mediated by the larger cues. Experiment 2 tested whether a different pattern of results would emerge with attentional cues that had been successfully employed to elicit a differential attentional effect on search performance as a function of cue size (e.g., Greenwood \& Parasuraman, 2004).

\section{EXPERIMENT 2 Spatial Characteristics of the Attentional Cue}

As in Experiment 1, here we also tested the hypothesis that the size of the attentional cue can differentially affect spatial resolution at the attended location, but in this experiment we used a different attentional cue. The texture display was similar to that of Experiment 1, but the attentional cue was composed of a frame presented around the to-be-attended area. Greenwood and Parasuraman (2004) employed similar frames as attentional cues and were able to successfully demonstrate a differential attentional effect on search performance due to the frame size. Hence, if large attentional cues lead to resolution decrement rather than enhancement, large frame-cues should improve performance at central locations and hinder performance at more peripheral locations. Alternatively, were the findings of Experiment 1 not specific to the cues employed, attentional effects on spatial resolution would only be found with small frame-cues, and we could conclude that transient attention cannot adapt its operation on spatial resolution on the basis of the scale of the cue.

\section{Method}

Observers. Twenty-four undergraduate students from the University of Haifa who did not participate in Experiment 1 participated in this experiment. All had normal or corrected-to-normal vision and were naive as to the purpose of the study.

Stimuli and Apparatus. The apparatus, texture, and mask displays were identical to those in Experiment 1. The attentional informative cue was a green $\left(43 \mathrm{~cd} / \mathrm{m}^{2}\right)$ rectangular frame created using a green $0.1^{\circ}$ line. The height of the frame was either $0.9^{\circ}$ - similar to the height of a single line element (Cue Size 1) - or $2.4^{\circ}$ - similar to the height of the texture target (Cue Sizes 3-15). The width of the frame varied systematically, as in Experiment 1, between $1\left(0.9^{\circ}\right.$ similar to a width of 1 column) to $15\left(10^{\circ}\right.$ - similar to a width of 15 columns). The noninformative cue consisted of a $7^{\circ} \times 29^{\circ}$ green frame surrounding the whole texture display. The center of the frame was aligned with the central row of the texture display.

Table 1

Eccentricity (in Degrees of Visual Angle) at Which the Performance Peak Occurred for the Noninformative and Informative Cues, Sizes 6-15, in Experiments 1-3

\begin{tabular}{ccccc}
\hline & \multicolumn{3}{c}{ Cue Size } & \\
\cline { 2 - 4 } Experiment & 6 & 9 & 15 & Noninformative \\
\hline 1 & 6.38 & 6.79 & 6.38 & 5.75 \\
2 & 5.26 & 4.71 & 5.19 & 5.49 \\
3 & 6.78 & 6.61 & 7.00 & 6.54 \\
\hline
\end{tabular}

Procedure. The procedure was identical to that of Experiment 1, with the following exceptions: In each block, half of the trials were preceded by a cue of a specific size, and half were preceded by a noninformative cue. Each observer performed between 100 and 160 practice trials, followed by 1,680 experimental trials divided into five blocks.

\section{Results and Discussion}

As in the previous experiment, the data were subjected to a within-observers three-way ANOVA (cue type $x$ cue size $\times$ target eccentricity) that revealed a significant three-way interaction $[F(24,552)=1.65, p<.05]$. For each cue size, the accuracy data were fitted to secondorder polynomials for the noninformative and informative conditions (Figure 3). Because in this experiment the numbers of informative and noninformative trials were the same, there was no need to combine data across block conditions. The data were further subjected to a withinobservers two-way ANOVA (cue type $\times$ target eccentricity) for each cue size. Here too, we replicated the central attentional impairment found with the smallest attentional cue. A significant cue type $\times$ target eccentricity interaction emerged for the smallest attentional cue, so that attention degraded performance at central locations and improved it at peripheral locations $[F(6,138)=3.340, p<$ .005]. This central attentional impairment, however, did not emerge with any other cue size. Although a significant interaction also emerged for Cue Size 3, it only stemmed from an attentional benefit at near eccentricities, without any distinctive cost at peripheral locations $[F(6,138)=$ $2.45, p<.05]$. This attentional benefit is also reflected in a significant main effect of cuing condition at Cue Size 3; as in Experiment 1, accuracy was higher in the informative than in the noninformative condition $[F(1,21)=6.83$, $p<.01]$. An analysis of RTs confirmed that no speedaccuracy trade-offs took place. In addition, as in Experiment 1 , there was no consistent difference for the different cues in the eccentricity at which performance peaked, including for the noninformative cue (Table 1). In sum, an attentional effect on spatial resolution was only found with small frame-cues. There was no effect on resolution, even with large frame-cues that previously had been used with sustained attention (e.g., Greenwood \& Parasuraman, 2004). These findings indicate that observers were not able to adjust the operation of transient attention in accordance with cue size. In Experiment 3, we tested whether a longer ISI between the cue and texture would enable transient attention to be more adaptable.

\section{EXPERIMENT 3 \\ Temporal Characteristics of the Attentional Cue}

Given that the time between cue onset and texture onset in Experiments 1 and 2 was derived from previous estimates of transient attentional shifts, it was presumably optimal for transient attention to focus on the cued region (see, e.g., Bergen \& Julesz, 1983; Nakayama \& Mackeben, 1989; Saarinen \& Julesz, 1991; Sagi \& Julesz, 1987; Tsal, 1983). Nevertheless, to ensure that the interval between the cue and the texture was not too short for 
transient attention to optimally adjust its operation on spatial resolution, in the present experiment we doubled the length of this interval, such that the cue would still be effective (Cheal \& Lyon, 1992; Nakayama \& Mackeben, 1989 ) yet eye movements still could not take place (Mayfrank et al., 1987).

\section{Method}

Observers. Thirty undergraduate students from the University of Haifa who did not participate in the previous experiments participated in Experiment 3; all had normal or corrected-to-normal vision and were naive as to the purpose of the study.

Stimuli, Apparatus, and Procedure. The stimuli, apparatus, and procedure were identical to those in Experiment 2, except that the length of the ISI between the cue and texture was doubled to $94 \mathrm{msec}$.

\section{Results and Discussion}

As in previous experiments, the data were subjected to a within-observers three-way ANOVA (cue type $\times$ cue size $\times$ target eccentricity) that revealed a significant three-way interaction $[F(24,696)=2.02, p<.005]$. The accuracy data for each cue size were fitted to second-order polynomials (Figure 4), and a within-observers two-way ANOVA (cue type $\times$ target eccentricity) was performed separately for each cue size. As in Experiments 1 and 2 and our previous findings (Yeshurun \& Carrasco, 1998), a significant cue type $\times$ target eccentricity interaction emerged for the smallest attentional cue: Attention degraded performance at central locations and improved it at peripheral locations $[F(6,174)=4.65, p<.0001]$. These findings suggest that the attentional effects on texture segmentation are robust and can be extended to a considerably longer cue-target ISI. A significant interaction also emerged for Cue Size $6[F(6,174)=5.76, p<.0001]$. The explanation for this interaction with Cue Size 6 is not clear, and it was not replicated in the other two experiments. There was no significant interaction with any other cue size. Thus, although occasionally such a cue type $\times$ target eccentricity interaction was found with cue sizes larger than 1 (i.e., Cue Size 9 in Experiment 1 and Cue Size 6 in this experiment), the only consistent interaction found across experiments was the interaction with the smallest cue size. Most importantly, as is clearly evi- dent in Figure 4 and Table 1, the pattern of the attentional effect on texture segmentation did not change gradually with large cues, even though the cue-target ISI here was twice as long as in the previous experiments. The analysis of correct RT data confirmed the absence of any speedaccuracy trade-offs.

In summary, even when a longer time was available for observers to deploy transient attention over larger areas, no evidence was found of lowered spatial resolution with larger attentional cues. This result seems consistent with a previous visual search study showing that doubling the ISI (from 60 to $120 \mathrm{msec}$ ) between cue and display did not yield a larger attentional benefit (Carrasco \& Yeshurun, 1998, Experiment 4).

\section{GENERAL DISCUSSION}

The goal of this study was to investigate the adaptability of transient attention regarding spatial resolution: Can the size of the attentional cue modulate the effect of transient attention on spatial resolution? The attentional cues we used were bars of different lengths in Experiment 1 and frames of different sizes in Experiments 2-3. The findings consistently replicated the attentional enhancement of spatial resolution reported previously with a small cue and a similar texture segmentation task (Carrasco et al., 2006; Talgar \& Carrasco, 2002; Yeshurun \& Carrasco, 1998), but there was no evidence of gradual resolution decrement with large cues. This was the case even when the cue-target ISI was doubled, to ensure enough available time for transient attention to adapt its operation on the basis of cue size (Experiment 3). Moreover, even if we only compared performance in the conditions in which cue size was larger than the target (i.e., Cue Sizes 6-15), there was no evidence of a resolution decrement with large cues. Had resolution gradually decreased as cue size gradually increased from 6 to 15 (i.e., cue sizes larger than the target), the eccentricity at which performance peaked would have gradually shifted to nearer eccentricities. However, as is evident in Table 1, there was no consistent difference in the peak eccentricities of these different cues. In fact, in all three experiments, performance with Cue Sizes 6-15 and with the noninformative cue peaked at around $6^{\circ}$ of eccentricity, which is in line with

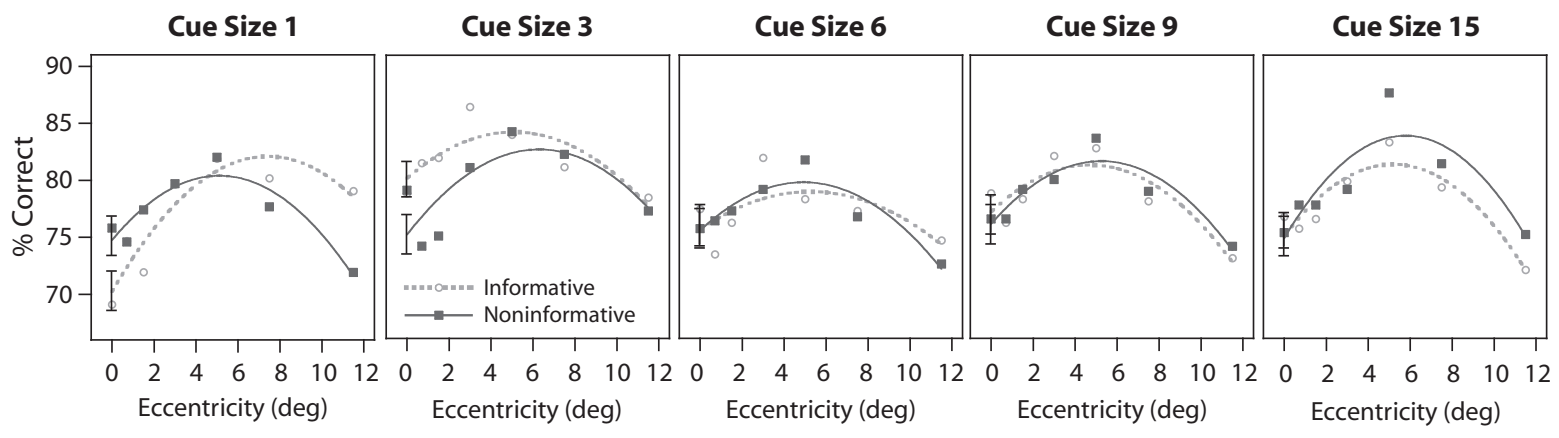

Figure 3. Observers' accuracy in Experiment 2 as a function of cue size and target eccentricity. Error bars correspond to one averaged standard error. 

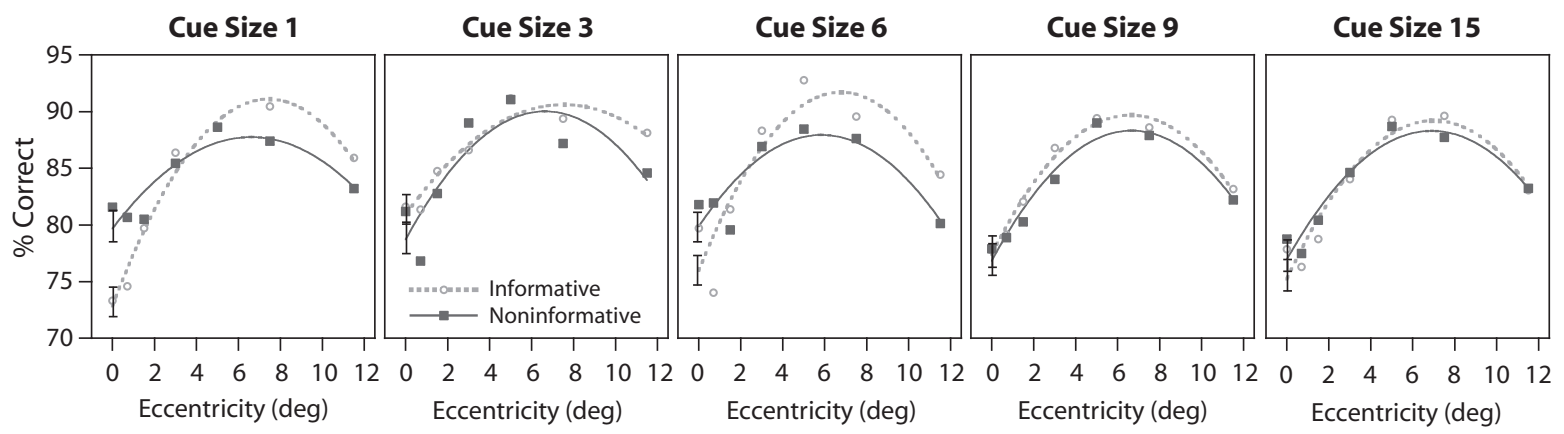

Figure 4. Observers' accuracy in Experiment 3 as a function of cue size and target eccentricity. Error bars correspond to one averaged standard error.

previous studies that examined the central performance drop with textures of scales similar to the one we employed here, but with no manipulation of spatial attention (e.g., Joffe \& Scialfa, 1995; Kehrer, 1989).

The finding that transient attention does not modulate its effect on spatial resolution on the basis of the size of the attentional cue might be due to the more automatic nature of transient attention. A growing body of evidence has demonstrated that transient attention is activated in a fast, automatic manner, and most likely operates at a relatively early stage of visual cortical processing (e.g., Cheal \& Lyon, 1991; Jonides, 1981; H. J. Müller \& Rabbitt, 1989; Nakayama \& Mackeben, 1989; Posner, 1980; Remington, Johnston, \& Yantis, 1992). For instance, in an orientation discrimination task, observers' contrast sensitivity was altered even when the validity of the transient cue was only $50 \%$ and the display consisted of two Gabor patches. Despite the fact that observers were told that the cue indicated the target location on only $50 \%$ of the trials and provided no information regarding the target orientation, and despite the simplicity of the display, the cue increased sensitivity at the cued location and impaired it at the uncued location, as compared with a central cue that did not indicate any location (Carrasco, Ling, \& Read, 2004; Pestilli \& Carrasco, 2005).

Moreover, the respective automaticity and flexibility of exogenous and endogenous cues has been evaluated using a response signal speed-accuracy trade-off (SAT) procedure. The results indicated that with endogenous cues, the observed attentional effects increased with cue validity. However, with exogenous cues, the attentional effects in both discriminability and processing speed were comparable across the range of cue validities. These results provide compelling time course evidence that transient attention is automatic (Giordano, McElree, \& Carrasco, 2004).

Like the present study, the studies just discussed illustrate the lack of flexibility of transient attention. That is, they all exemplify cases in which granting priority to the information at the attended location over the information at other locations has not necessarily resulted in optimal performance. The fact that transient attention is fast and does not require conscious processing allows us to deal successfully with unexpected events occurring outside the focus of our conscious interest. This could be crucial for our survival. Still, the present findings suggest that the "price" of the fast and automatic nature of transient attention is that it is a more rigid mechanism that cannot flexibly operate in both directions - resolution enhancement and decrement - at least not as a function of the scale of the attention-attracting information. We are currently investigating whether manipulating other aspects of the visual display will enable transient attention to affect perception in a more flexible way, as well as whether the more controlled nature of sustained attention makes it a more flexible mechanism that can either increase or decrease spatial resolution.

Interestingly, in Experiments 1 and 2 an attentional benefit emerged for Cue Size 3. This benefit most likely stems from the fact that the width of Cue Size 3 matched the width of the target, but at this time we can only speculate regarding the specific mechanism underlying this benefit. Perhaps, when the cue equaled the target size, attention was drawn to the borders between the target and background, facilitating segregation regardless of the spatial resolution at the target eccentricity. Further research is required to understand the processes underlying this benefit. For instance, a simultaneous manipulation of the sizes of the target and cue, as well as a manipulation of the degree of uncertainty regarding the size of the target, might help us better understand the source of this attentional benefit.

Finally, consistent attentional effects were found only with Cue Sizes 1 and 3, in which there was no spatial uncertainty regarding the target location. Some authors have attributed attentional effects to the fact that a cue reduces spatial uncertainty. They suggest that the attentional precue allows observers to monitor only the relevant location rather than all possible ones, leading to a reduction of the statistical noise introduced at the irrelevant locations (e.g., Davis, Kramer, \& Graham, 1983; Eckstein, 1998; Foley \& Schwarz, 1998; Kinchla, 1980; Palmer, 1994; Shaw, 1984; Solomon, Lavie, \& Morgan, 1997; Sperling \& Dosher, 1986). Indeed, uncertainty reduction plays an important role in many tasks and should be used as a benchmark against which to compare attentional effects. However, it has been shown that the effects of attention go beyond uncertainty reduction. For instance, significant attentional 
effects on spatial resolution and contrast sensitivity have been found even under conditions of negligible spatial uncertainty - with a suprathreshold target, presented in isolation, either followed or not by a local postmask (see, e.g., Carrasco et al., 2000; Carrasco et al., 2002; Ling \& Carrasco, 2006; Yeshurun \& Carrasco, 1999); in addition, the precuing effect found with briefly presented orientation displays is greater than that predicted from the signal detection model of spatial uncertainty (Morgan, Ward, \& Castet, 1998); and finally, a mere reduction in spatial uncertainty cannot account for the way in which spatial visual thresholds are influenced by the near absence of attention (Lee, Itti, Koch, \& Braun, 1999).

Given that reduced spatial uncertainty always predicts improved performance, it cannot explain the central attentional impairment elicited by a small cue reported here and in our previous studies (Carrasco et al., 2006; Talgar \& Carrasco, 2002; Yeshurun \& Carrasco, 1998, 2000). Moreover, from the spatial uncertainty reduction account, one would predict a gradually increasing effect of attention as uncertainty is gradually reduced. However, none of the experiments showed such a gradual change in performance as a function of cue size. For instance, with Cue Size 6, the $3 \times 3$ target would appear within a $3 \times 6$ region. The degree of uncertainty associated with this cue size was greatly reduced relative to the noninformative cue, which indicated that the target will appear within a $3 \times 41$ region. Yet this significant reduction in spatial uncertainty did not result in any reliable performance advantage or disadvantage for Cue Size 6 as compared with the noninformative condition.

To conclude, systematic manipulation of the size of the attentional cue demonstrated a differential effect for the different cue sizes, but this differential effect merely reflected an attentional enhancement of spatial resolution with small cues, but no effect on resolution with large cues. That is, transient attention enhances spatial resolution at the attended location when it is attracted to that location by a small cue but does not lower resolution when it is attracted by a large cue. Thus, transient attention cannot adapt its operation on spatial resolution on the basis of the size of the attentional cue.

\section{AUTHOR NOTE}

This research was supported by Grant 2001169 from the United States-Israel Binational Science Foundation (BSF), Jerusalem, Israel, to Y.Y. and M.C. We thank Stuart Fuller, Anna Marie Giordano, Taosheng Liu, Sam Ling, Barbara Montagna, and the referees for helpful comments on drafts of the manuscript. Correspondence relating to this article may be sent to Y. Yeshurun, Department of Psychology, University of Haifa, Haifa, 31905 Israel (e-mail: yeshurun@research.haifa.ac.il).

\section{REFERENCES}

BaLz, G. W., \& HocK, H. S. (1997). The effect of attentional spread on spatial resolution. Vision Research, 37, 1499-1510.

Bergen, J. R., \& Julesz, B. (1983). Parallel versus serial processing in rapid pattern discrimination. Nature, 303, 696-698.

Carrasco, M., Evert, D. L., Chang, I., \& Katz, S. M. (1995). The eccentricity effect: Target eccentricity affects performance on conjunction searches. Perception \& Psychophysics, 57, 1241-1261.

Carrasco, M., \& Frieder, K. S. (1997). Cortical magnification neutralizes the eccentricity effect in visual search. Vision Research, 37, 63-82.
Carrasco, M., Ling, S., \& Read, S. (2004). Attention alters appearance. Nature Neuroscience, 7, 308-313.

Carrasco, M., Loula, F., \& Ho, Y.-X. (2006). How attention enhances spatial resolution: Evidence from selective adaptation to spatial frequency. Perception \& Psychophysics, 68, 1004-1012.

Carrasco, M., McLean, T. L., Katz, S. M., \& Frieder, K. S. (1998). Feature asymmetries in visual search: Effects of display duration, target eccentricity, orientation and spatial frequency. Vision Research, 38, 347-374.

Carrasco, M., Penpeci-Talgar, C., \& Eckstein, M. (2000). Spatial covert attention increases contrast sensitivity across the CSF: Support for signal enhancement. Vision Research, 40, 1203-1215.

Carrasco, M., Williams, P. E., \& Yeshurun, Y. (2002). Covert attention increases spatial resolution with or without masks: Support for signal enhancement. Journal of Vision, 2, 467-479.

Carrasco, M., \& Yeshurun, Y. (1998). The contribution of covert attention to the set-size and eccentricity effects in visual search. Journal of Experimental Psychology: Human Perception \& Performance, 24, 673-692.

Castiello, U., \& Umiltà, C. (1990). Size of the attentional focus and efficiency of processing. Acta Psychologica, 73, 195-209.

Cheal, M., \& Lyon, D. R. (1991). Central and peripheral precuing of forced-choice discrimination. Quarterly Journal of Experimental Psychology, 43A, 859-880.

CheAL, M., \& LyON, D. R. (1992). Benefits from attention depend on the target type in location-precued discrimination. Acta Psychologica, 81, 243-267.

Cohen, J. D., MacWhinney, B., Flatt, M., \& Provost, J. (1993). PsyScope: An interactive graphic system for designing and controlling experiments in the psychology laboratory using Macintosh computers. Behavior Research Methods, Instruments, \& Computers, 25, 257-271.

Corbetta, M., \& Shulman, G. L. (2002). Control of goal-directed and stimulus-driven attention in the brain. Nature Reviews Neuroscience, 3, 201-215.

Davis, E. T., Kramer, P., \& Graham, N. (1983). Uncertainty about spatial frequency, spatial position, or contrast of visual patterns. Perception \& Psychophysics, 33, 20-28.

Desimone, R., \& Duncan, J. (1995). Neural mechanisms of selective visual attention. Annual Review Neuroscience, 18, 193-222.

De Valois, R. L., \& De Valois, K. K. (1988). Spatial vision. New York: Oxford University Press.

Eckstein, M. P. (1998). The lower efficiency for conjunctions is due to noise and not serial attentional processing. Psychological Science, 9, 111-118.

ERIKSEN, C. W. (1990). Attentional search of the visual field. In D. Brogan (Ed.), Visual search (pp. 3-19). London: Taylor \& Francis.

ERIKSEN, C. W., \& ST. JAMES, J. D. (1986). Visual attention within and around the field of focal attention: A zoom lens model. Perception \& Psychophysics, 40, 225-240.

Eriksen, C. W., \& YeH, Y. (1985). Allocation of attention in the visual field. Journal of Experimental Psychology: Human Perception \& Performance, 11, 583-597.

Foley, J. M., \& Schwarz, W. (1998). Spatial attention: Effect of position uncertainty and number of distractor patterns on the thresholdversus-contrast function for contrast discrimination. Journal of the Optical Society of America A, 15, 1036-1047.

Giordano, A. M., McElree, B., \& Carrasco, M. (2004). On the automaticity and flexibility of covert attention [Abstract]. Journal of Vision, 4(8), 627a.

Golla, H., Ignashchenkova, A., Haarmeier, T., \& Thier, P. (2004). Improvement of visual acuity by spatial cueing: A comparative study in human and non-human primates. Vision Research, 44, 1589-1600.

Goto, M., Toriu, T., \& Tanahashi, J. (2001). Effect of size of attended area on contrast sensitivity function. Vision Research, 41, 1483-1487.

Graham, N. V. S. (1989). Visual pattern analyzers. New York: Oxford University Press.

Greenwood, P. M., \& Parasuraman, R. (2004). The scaling of spatial attention in visual search and its modification in healthy aging. Perception \& Psychophysics, 66, 3-22.

Gurnsey, R., Pearson, P., \& Day, D. (1996). Texture segmentation along the horizontal meridian: Nonmonotonic changes in perfor- 
mance with eccentricity. Journal of Experimental Psychology: Human Perception \& Performance, 22, 738-757.

Hock, H. S., Balz, G. W., \& Smollon, W. (1998). Attentional control of spatial scale: Effects on self-organized motion patterns. Vision Research, 38, 3743-3758.

HüBNER, R. (2000). Attention shifting between global and local target levels: The persistence of level-repetition effects. Visual Cognition, 7, 465-484.

Joffe, K. M., \& Scialfa, C. T. (1995). Texture segmentation as a function of eccentricity, spatial frequency, and target size. Spatial Vision, 9, 325-342.

JoNIDES, J. (1981). Voluntary vs. automatic control over the mind's eye's movement. In J. Long \& A. Baddeley (Eds.), Attention and performance IX (pp. 187-204). Hillsdale, NJ: Erlbaum.

KeHRER, L. (1989). Central performance drop on perceptual segregation tasks. Spatial Vision, 4, 45-62.

KeHRER, L. (1997). The central performance drop in texture segmentation: A simulation based on spatial filter model. Biological Cybernetics, 77, 297-305.

Kinchla, R. A. (1980). The measurement of attention. In R. S. Nickerson (Ed.), Attention and performance VIII (pp. 213-238). Hillsdale, NJ: Erlbaum.

Kinchla, R. A., Solis-Macias, V., \& Hoffman, J. (1983). Attending to different levels of structure in a visual image. Perception \& Psychophysics, 33, 1-10.

LaBerge, D., Brown, V., Carter, M., Bash, D., \& Hartley, A. (1991). Reducing the effects of adjacent distractors by narrowing attention. Journal of Experimental Psychology: Human Perception \& Performance, 17, 65-76.

LAMB, M. R., \& YUnd, E. W. (1996). Spatial frequency and attention: Effects of level-, target-, and location-repetition on the processing of global and local forms. Perception \& Psychophysics, 58, 363-373.

Lee, D. K., Itti, L., Косн, C., \& Braun, J. (1999). Attention activates winner-take-all competition among visual filters. Nature Neuroscience, 2, 375-381.

Ling, S., \& Carrasco, M. (2006). Sustained and transient covert attention enhance the signal via different contrast response functions. Vision Research, 46, 1210-1220.

Lu, Z. L., \& Dosher, B. A. (1998). External noise distinguishes attention mechanisms. Vision Research, 38, 1183-1198.

Luck, S. J., Chelazzi, L., Hillyard, S. A., \& Desimone, R. (1997). Neural mechanisms of spatial selective attention in areas V1, V2, and V4 of macaque visual cortex. Journal of Neurophysiology, 77, 24-42.

MaYfrank, L., Kimmig, H., \& Fischer, B. (1987). The role of attention in the preparation of visually guided saccadic eye movements in man. In J. K. O’Regan \& A. Lévy-Schoen (Eds.), Eye movements: From physiology to cognition (pp. 37-45). Amsterdam: North-Holland.

Moran, J., \& Desimone, R. (1985). Selective attention gates visual processing in the extrastriate cortex. Science, 229, 782-784.

Morgan, M. J., WARD, R. M., \& CASTET, E. (1998). Visual search for a tilted target: Tests of spatial uncertainty models. Quarterly Journal of Experimental Psychology, 51A, 347-370.

MülleR, H. J., \& RabbitT, P. M. A. (1989). Reflexive and voluntary orienting of visual attention: Time course of activation and resistance to interruption. Journal of Experimental Psychology: Human Perception \& Performance, 15, 315-330.

Müller, N. G., Bartelt, O. A., Donner, T. H., Villringer, A., \& BrandT, S. A. (2003). A physiological correlate of the "zoom lens" of visual attention. Journal of Neuroscience, 23, 3561-3565.

Nakayama, K., \& Mackeben, M. (1989). Sustained and transient components of focal visual attention. Vision Research, 29, 1631-1646.

Palmer, J. (1994). Set-size effects in visual search: The effect of attention is independent of the stimulus for simple tasks. Vision Research, 34, 1703-1721.

Pestilli, F., \& Carrasco, M. (2005). Contrast sensitivity is enhanced at cued and impaired at uncued locations. Vision Research, 45, 1867-1875.

Phillips, G. C., \& Wilson, H. R. (1984). Orientation bandwidths of spatial mechanisms measured by masking. Journal of the Optical Society of America A, 1, 226-232.

Posner, M. I. (1980). Orienting of attention. Quarterly Journal of Experimental Psychology, 32, 3-25.

PoteChin, C., \& GURNSEY, R. (2003). Backward masking is not required to elicit the central performance drop. Spatial Vision, 16, 393-406.

Remington, R. W., Johnston, J. C., \& YAnTis, S. (1992). Involuntary attentional capture by abrupt onsets. Perception \& Psychophysics, 51, 279-290.

Reynolds, J. H., \& ChelazzI, L. (2004). Attentional modulation of visual processing. Annual Review of Neuroscience, 27, 611-647.

REYNOLDS, J. H., \& DESIMONE, R. (1999). The role of neural mechanisms of attention in solving the binding problem. Neuron, 24, 19-25.

Robertson, L. C., Egly, R., Lamb, M. R., \& Kerth, L. (1993). Spatial attention and cuing to global and local levels of hierarchical structure. Journal of Experimental Psychology: Human Perception \& Performance, 19, 471-487.

Rosen, A. C., Rao, S. M., Caffarra, P., Scaglioni, A., Bobholz, J. A., Woodley, S. J., ET AL. (1999). Neural basis of endogenous and exogenous spatial orienting: A functional MRI study. Journal of Cognitive Neuroscience, 11, 135-152.

SaARINEn, J., \& Julesz, B. (1991). The speed of attentional shifts in the visual field. Proceedings of the National Academy of Sciences, 88, $1812-1814$

SAGI, D., \& Julesz, B. (1987). Short-range limitation on detection of feature differences. Spatial Vision, 2, 39-49.

SHAw, M. L. (1984). Division of attention among spatial locations: A fundamental difference between detection of letters and detection of luminance increments. In H. Bouma \& D. G. Bouwhuis (Eds.), Attention and performance X: Control of language processes (pp. 109-120). Hillsdale, NJ: Erlbaum

Shulman, G. L., \& Wilson, J. (1987). Spatial frequency and selective attention to local and global information. Perception, 16, 89-101.

Solomon, J. A., Lavie, N., \& Morgan, M. J. (1997). Contrast discrimination function: Spatial cuing effects. Journal of the Optical Society of America A, 14, 2443-2448.

Sperling, G., \& Dosher, B. A. (1986). Strategy and optimization in human information processing. In K. R. Boff, L. Kaufman, \& J. P. Thomas (Eds.), Handbook of perception and human performance (Vol. 1, chap. 2, pp. 1-65). New York: Wiley.

TAlgar, C. P., \& CARRASCO, M. (2002). Vertical meridian asymmetry in spatial resolution: Visual and attentional factors. Psychonomic Bulletin \& Review, 9, 714-722.

Tsal, Y. (1983). Movements of attention across the visual field. Journal of Experimental Psychology: Human Perception \& Performance, 9 , 523-530.

WARD, L. M. (1982). Determinants of attention to local and global features of visual forms. Journal of Experimental Psychology: Human Perception \& Performance, 8, 562-581.

YANTIS, S. (1996). Attentional capture in vision. In A. F. Kramer, M. G. H. Coles, \& G. D. Logan (Eds.), Converging operations in the study of visual selective attention (pp. 45-76). Washington, DC: American Psychological Association.

YeshuRUn, Y., \& CARRASCO, M. (1998). Attention improves or impairs visual performance by enhancing spatial resolution. Nature, 396, 72-75.

Yeshurun, Y., \& CARRasco, M. (1999). Spatial attention improves performance in spatial resolution tasks. Vision Research, 39, 293-306.

Yeshurun, Y., \& Carrasco, M. (2000). The locus of attentional effects in texture segmentation. Nature Neuroscience, 3, 622-627.

(Manuscript received November 28, 2005; revision accepted for publication July 9, 2007.) 